\title{
Simultaneous acoustic radiation force imaging and MR thermometry based on a coherent echo-shifted sequence
}

\author{
Yangzi Qiao ${ }^{1,2 \#}$, Chao Zou ${ }^{1,2 \#}$, Chuanli Cheng ${ }^{1}$, Changjun Tie ${ }^{1}$, Qian Wan ${ }^{1,3}$, Hao Peng ${ }^{1,4}$, Dong Liang ${ }^{1}$, \\ Xin Liu ${ }^{1,2}$, Hairong Zheng ${ }^{1,2}$ \\ ${ }^{1}$ Paul C. Lauterbur Research Center for Biomedical Imaging, Shenzhen Institutes of Advanced Technology, Chinese Academy of Sciences, Shenzhen, \\ China; ${ }^{2}$ Shenzhen Key Laboratory of Ultrasound Imaging and Therapy, Shenzhen, China; ${ }^{3}$ Institute of Biomedical and Health Engineering, \\ Shenzhen College of Advanced Technology, University of Chinese Academy of Sciences, Shenzhen, China; ${ }^{4}$ Key Laboratory of Imaging Processing \\ and Intelligence Control, School of Artificial Intelligence and Automation, Huazhong University of Science and Technology, Wuhan, China
}

"These authors contributed equally to this work.

Correspondence to: Dr. Hairong Zheng. Paul C. Lauterbur Research Center for Biomedical Imaging, Shenzhen Institutes of Advanced Technology, Chinese Academy of Sciences, 1068 Xueyuan Avenue, Shenzhen University Town, Shenzhen, China. Email: hr.zheng@siat.ac.cn.

Background: Simultaneous magnetic resonance (MR) acoustic radiation force imaging (ARFI) and MR thermometry (MRT) (STARFI) based on coherent echo-shifted (cES) sequence was proposed and comprehensively compared to radiofrequency (RF)-spoiled gradient echo (spGRE) STARFI.

Methods: Through use of delicately designed gradients, a collection of echoes was delayed by one repetition time (TR) cycle. The crusher gradient after readout (RO) was used as the displacement encoding gradient (DEG). The sequence was intrinsically sensitive to temperature. High-intensity focused ultrasound (HIFU) pulses were interleaved ON/OFF in successive TRs to separate the phase changes induced by displacement due to acoustic radiation force (ARF) impulses and temperature. Bloch simulation was performed to study the phase sensitivity to displacement of the proposed cES STARFI and spGRE STARFI. The proposed cES sequence was evaluated and compared to spGRE STARFI in ex vivo porcine muscle and ex vivo porcine brain.

Results: The minimally achievable TR of cES STARFI was shorter than that of spGRE STARFI, indicating that the cES sequence was more time efficient. It was verified through Bloch simulation and $e x$ vivo experiments that the phase sensitivity to displacement of cES STARFI was higher than that of spGRE STARFI. The optimal trigger delays of cES STARFI and spGRE STARFI in $e x$ vivo porcine muscle were $t_{\text {offset }}=-2$ and $-1 \mathrm{~ms}$, respectively. The displacement-induced phase change to acoustic pressure slopes of cES STARFI were $0.079,0.079$, and $0.047 \mathrm{rad} / \mathrm{Mpa}$ across the three muscle samples, while the slopes of spGRE STARFI were only $0.047,0.052$, and $0.027 \mathrm{rad} / \mathrm{Mpa}$. The maximum temperature difference between cES STARFI and spGRE STARFI was $1.1^{\circ} \mathrm{C}$. In $e x$ vivo porcine brain, both the displacement-induced phaseto-noise ratio (PNRd) and the temperature uncertainty of cES STARFI were better than those of spGRE STARFI $(\mathrm{P}<0.05)$. The temperature and displacement-induced phase change maps of cES STARFI and spGRE STARFI during HIFU treatment were in good accordance in time and spatial location.

Conclusions: The cES STARFI sequence can provide simultaneous MR-ARFI and temperature measurements during pulsed HIFU applications. Though the exact displacement cannot be quantified directly, the sequence showed increased phase sensitivity compared with the spGRE sequence and provided efficient visualization of the focal spot. cES STARFI could therefore be a desirable alternative to spGRE STARFI in practical applications.

Keywords: High-intensity focused ultrasound (HIFU); acoustic radiation force imaging (ARFI); magnetic resonance thermometry (MRT); coherent echo-shifted sequence (cES sequence) 
Submitted Feb 13, 2020. Accepted for publication Jun 18, 2020.

doi: 10.21037/qims-20-274

View this article at: http://dx.doi.org/10.21037/qims-20-274

\section{Introduction}

High-intensity focused ultrasound (HIFU) is a noninvasive therapeutic treatment for several diseases, including uterine fibrosis (1), prostate cancer (2), and essential tremor (3). Treatment with HIFU should always be closely monitored due to safety concerns. Magnetic resonance imaging (MRI) is a widely used imaging guidance modality applied in HIFU treatment (4). One essential process in MRI-guided HIFU treatment is the localization of the ultrasound focus. This becomes more critical in transcranial applications, as ultrasound beams may deviate from the desired target. MR acoustic radiation force imaging (MR-ARFI) $(5,6)$ can encode micro-scale tissue displacements caused by millisecond-short impulses of HIFU into MR phase contrast. This can be used to localize the focus. Spatially distributed phase contrast information can also be used to correct transcranial phase aberrations $(7,8)$. During the treatment planning stage, MR-ARFI might be performed repeatedly and may induce unwanted heating at the brainskull interface. Therefore, it is crucial to simultaneously monitor the temperature to avoid significant temperature increases in brain tissue near the skull, which could cause thermal damage to other regions. Although MR-ARFI sequences could be incorporated with echo planar imaging (EPI) readouts (ROs) to reduce the number of focused ultrasound (FUS) impulses and prevent thermal risk (9), EPI images are vulnerable to off-resonance effects.

Simultaneous MR thermometry (MRT) imaging and MRARFI (STARFI) has been considered since 2012 (10-14). With the addition of temperature monitoring during focus localization in the treatment planning stage, acquiring both MR-ARFI and MRT images from a single sequence can ensure the comprehensively monitoring and evaluation of pulsed HIFU therapy; these images represent the mechanical and thermal effects, two of the most essential effects of therapeutic HIFU (15). Most simultaneous MRT and MR-ARFI has been based on radiofrequency (RF)spoiled gradient echo (spGRE) sequence, which is the most frequently used MRT sequence. Additional bipolar displacement encoding gradients (DEGs) were always added before the RO gradient. To separate the displacementinduced phase change from that induced by temperature, inverted bipolar DEGs should be used in another repetition time (TR) cycle $(10,13)$. However, this required additional correction for the eddy current effect. To avoid the eddy current effect induced by opposite DEG polarities, the use of DEGs with the same polarity has been considered for ARFI sequences. Mougenot et al. (12) proposed to alternate the start point of the HIFU pulse during every odd and even acquisition while keeping the polarity of DEG unchanged. This approach improved displacement map quality at the cost of dead time between the two DEG lobes. De Bever et al. (14) suggested acquiring images with ultrasound pulses $(\mathrm{ON})$ and without ultrasound pulses (OFF) during the DEG at successive TRs without inverting the DEG polarity. These approaches ensure that the eddy-current-induced phase change can be canceled out by subsequent phase subtraction. Even though spGRE STARFI can provide reliable results, many studies are still exploring new sequences that are more sensitive to subtle displacement or temperature change. Zheng et al. (16) proposed an MR-ARFI with increased phase sensitivity based on passband balanced steady-state free precession (SSFP). However, this increased sensitivity is limited to a narrow off-resonance range. Peng et al. (17) demonstrated that echo-shifted (ES) sequence has better temperature uncertainty performance compared with spGRE sequence with the same temporal resolution, especially for tissue with longer T2/T2*. However, little attention has been paid to the ARFI sensitivity of ES sequence.

ES sequence was first introduced by Moonen et al. (18) to increase $\mathrm{T}^{*}$ sensitivity while maintaining a short TR. By adding crusher gradients, echo formation is delayed by one or more TR cycles, allowing echo time (TE) to be greater than TR. The prolonged TE increases temperature sensitivity and precision efficiency (17) compared with the more commonly used spGRE sequence. The additional crusher gradient also makes the sequence intrinsically motion sensitive. These ES characteristics make it an attractive option for simultaneous ARFI and temperature imaging.

In this study, a coherent echo-shifted (cES STARFI) sequence was proposed to simultaneously monitor the thermal and mechanical effects of HIFU treatment. Without RF spoiling, it was assumed that the multiple- 
pathway contributions of phase accumulation would increase the phase sensitivity to displacement caused by acoustic radiation force (ARF) impulse. The performance of cES STARFI was carefully compared with spGRE STARFI using Bloch simulation and ex vivo experiments on porcine muscle and brain samples.

\section{Methods}

\section{Sequences}

The timing diagrams of the cES STARFI and spGRE STARFI sequences are shown in Figure $1 A, B . M_{c r u}$ is the net unbalanced gradient moment of the cES sequence within each TR. The $M_{c r u}$ should be large enough to produce artifact-free images (19). The unbalanced gradient can be added in any of three directions. In Figure 1A, this was added in the slice selection (SS) direction as an example. Gradients $\mathrm{C}$ and D were designed so that echo collection could be delayed by one TR. The gradient moments should satisfy the following:

$$
\begin{aligned}
& M_{C}=-M_{c r u} \\
& M_{D}=2 \times M_{c r u}-M_{A} / 2
\end{aligned}
$$

Gradient D was applied after the RO gradient and used for displacement encoding with synchronization to the HIFU pulse for the cES STARFI sequence. The duration of the HIFU pulse was equal to the duration of gradient D. In the comparison study, the duration and the amplitude of gradient $\mathrm{D}$ were kept the same for both sequences.

For both the cES and spGRE sequences, two echoes were interleaved in successive TRs with the HIFU pulse ON/OFF to avoid eddy current effects. Two images were collected as a pair. For cES STARFI, coherent echo was collected without RF phase cycling. Echo 1 was primarily sensitive to temperature, while Echo 2 was sensitive to both temperature and displacement. Comparatively, Echo 2 of spGRE STARFI was sensitive to temperature only, whereas Echo 1 was sensitive to both temperature and displacement. It was assumed that the temperature change between Echo 1 and Echo 2 (one pair) was negligible, as the $k$-space lines were acquired in an interleaved way and the time interval between the two lines of $k$-space was only one TR. The displacement-induced phase change could therefore be separated from that induced by temperature using phase subtraction between the two echoes. Four pairs of images were acquired before HIFU sonication to ensure that the steady state of MR signal had been reached. Images of pair \#4 were used as a reference for temperature calculations according to the following equations:

$$
\begin{aligned}
& \Delta T_{c E S}=\frac{\Delta \varphi_{T_{c E S}}}{\alpha 2 \pi \gamma B_{0} T E_{e f f}}=\frac{\varphi_{\text {echo }_{c E S}}-\varphi_{\text {refecho }}}{\alpha 2 \pi \gamma B_{0}(T E+T R)}
\end{aligned}
$$

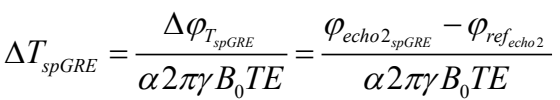

where, $\alpha\left(-0.01 \mathrm{ppm} /{ }^{\circ} \mathrm{C}\right)$ is the proton resonance frequency shift (PRFS) coefficient (20), $\gamma$ is the gyro-magnetic ratio, $\mathrm{B} 0$ is the main magnetic field strength, $\mathrm{TE}_{\text {eff }}$ is the effective TE of the cES STARFI sequence, and $\mathrm{TE}_{\text {eff }}=\mathrm{TR}+\mathrm{TE}(21)$. Tissue displacement $\mathrm{D}(\mathrm{t})$ induced by ARF impulse accumulated a phase change $\Delta \varphi_{\mathrm{D}}$ according to the following equation (22):

$$
\Delta \varphi_{D}=2 \pi \gamma \cdot \zeta \int_{0}^{\tau} G_{D E G}(t) D(t) d t
$$

In Eq. [5], $G_{D E G}(t)$ is the amplitude of the DEG, and the duration of the gradient is $\tau$. $\zeta$ is defined as the phase sensitivity to displacement induced by the ARF impulse. For traditional Spin Echo (SE)-ARFI and GRE-ARFI, $\zeta=1$. However, for multi-pathway sequences, $\zeta$ might be more than 1 due to historical phase contributions to the current echo. Therefore, the phase sensitivity $\zeta$ of the multipathway sequence depends on tissue properties (T1/T2) and imaging parameters. By ignoring the temperature difference between the two echoes, the displacement-induced phase change can be calculated as follows:

$$
\begin{aligned}
& \Delta \varphi_{D_{C E S}}=\varphi_{\text {echo } 2_{c E S}}-\varphi_{\text {echo } 1_{c E S}} \\
& \Delta \varphi_{D_{s p G R E}}=\varphi_{\text {echo } 1_{s p C R E}}-\varphi_{\text {echo } 2_{s p C R E}}
\end{aligned}
$$

\section{Bloch simulation}

Bloch simulation was carried out to determine the phase sensitivity of cES STARFI and spGRE STARFI to displacement $\zeta$ according to the following equation:

$$
\zeta=\frac{\Delta \varphi_{D}}{2 \pi \gamma G_{D E G} \tau D(t)}
$$

The input displacement was first set to a fixed value of $D(t)=5 \mu \mathrm{m}$, and the DEGs were simplified to a rectangle shape. The amplitudes of DEGs $\left(G_{D E G}\right)$ were all set to $50 \mathrm{mT} / \mathrm{m}$, the same as that used in the ex vivo experiments. Only the DEGs (C and D in Figure 1) were considered in the 

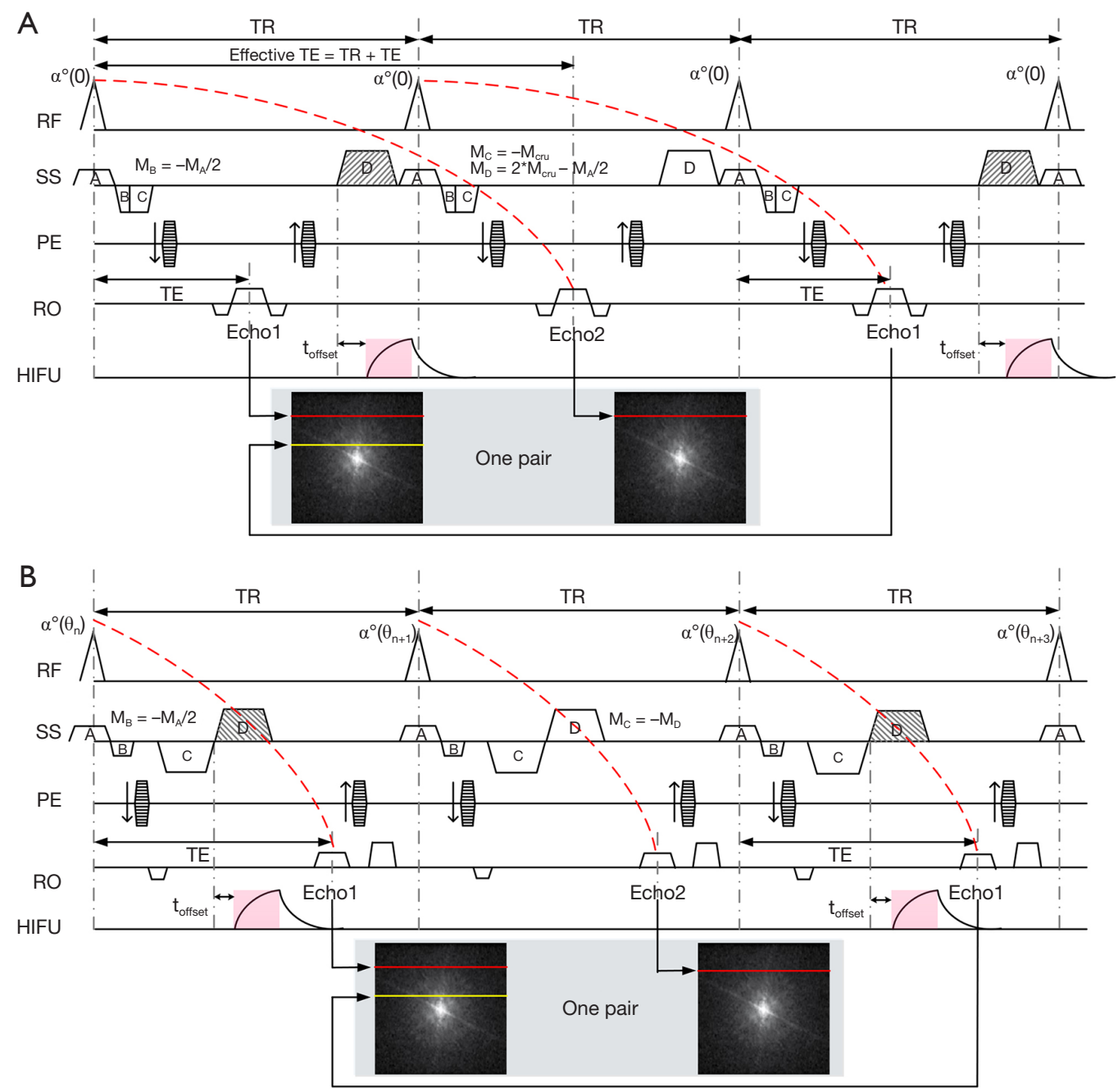

Figure 1 Timing diagram of the (A) cES STARFI and (B) spGRE STARFI sequences. A is the SS gradient, and B is the slice refocusing gradient in both sequences. Gradients $\mathrm{C}$ and $\mathrm{D}$ in (A) were designed to shift the echo to the next TR; gradient D also performed displacement encoding. $M_{A}, M_{B}, M_{c}$, and $M_{D}$ indicate moments of the corresponding gradients. Mcru is the net unbalanced gradient moment within each TR. Gradients C and D in (B) are the two lobes of bipolar DEG. The HIFU pulse is interleaved ON/OFF in successive TRs. The time offset between the start time point of the HIFU pulse and gradient D is the toffset. Data collected from Echo 1 and Echo 2 are grouped as a pair. cES, coherent echo-shifted; spGRE, spoiled gradient echo; TR, repetition time; DEG, displacement encoding gradient; HIFU, high-intensity focused ultrasound; TE, echo time; RF, radiofrequency; SS, slice selection; PE, phase encode; RO, readout.

simulation; the other imaging gradients (SS, phase encoding, and $\mathrm{RO}$ gradients) were omitted. The net unbalanced gradient moment of cES STARFI in each TR caused a phase dispersion of $44 \pi$ in the SS direction, corresponding to $M_{c}=-103.36 \mathrm{mT} / \mathrm{m} \cdot \mathrm{ms}$ and $M_{D}=206.72 \mathrm{mT} / \mathrm{m} \cdot \mathrm{ms}$ under a slice thickness of $5 \mathrm{~mm}$. The moments of gradients $\mathrm{C}$ and $D$ in spGRE STARFI were: $M_{c}=-M_{D}=-206.72 \mathrm{mT} / \mathrm{m} \cdot \mathrm{ms}$. The influences of T1/T2 on the phase sensitivity to displacement of cES STARFI and spGRE STARFI were explored, and a total of 300 isochromats and 600 TRs were simulated. The RF pulse was assumed to rotate all isochromats instantaneously. An incremental spoiler angle of $117^{\circ}$ and a crusher moment of $2 \pi$ were used in the spGRE STARFI simulation. Other simulation parameters were consistent with the ex vivo experiment: TE/TR $=6.4 / 14.3 \mathrm{~ms}$ for $\mathrm{cES}$ STARFI, and TE $/ \mathrm{TR}=13.3 / 16.9 \mathrm{~ms}$ for spGRE STARFI, both of which are the minimum TE/ TR that the sequences can reach. Flip angle $(\mathrm{FA})=10^{\circ}$, T1 


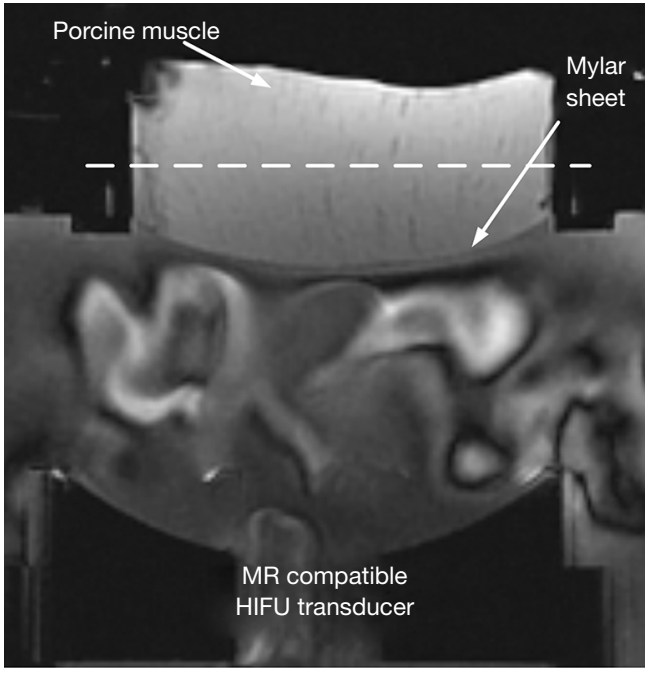

Figure 2 System setup. A $0.8 \mathrm{MHz}$ MR-compatible HIFU transducer was used to induce local displacement in ex vivo porcine muscle. The dashed line indicates the imaging plane. MR, magnetic resonance; HIFU, high-intensity focused ultrasound.

$=200-2,000 \mathrm{~ms}$ with a step of $100 \mathrm{~ms}$, and T2 $=10-190 \mathrm{~ms}$ with a step of $10 \mathrm{~ms}$. The impact of the displacementinduced phase change on the calculation of temperature was also simulated.

As the actual displacement was not achieved instantaneously, the displacement-induced phase change also depended on the start time of the HIFU pulse. The optimal trigger delays of cES STARFI and spGRE STARFI were simulated with $t_{\text {offset }}$ varied from -4 to $2 \mathrm{~ms}$ with a step of $0.5 \mathrm{~ms}$. The instantaneous tissue displacement at the focus was modeled based on overdamped harmonic response $(22,23)$ :

$$
\begin{aligned}
& D(t)=D_{0}\left(1-\exp \left(-\frac{t-t_{\text {offset }}}{\tau_{\text {rise }}}\right)\right), t_{\text {offset }} \leq t \leq t_{\text {off }} \\
& D(t)=D\left(t_{\text {off }}\right) \exp \left(-\frac{t-t_{\text {offset }}}{\tau_{\text {fall }}}\right), t_{\text {off }}<t
\end{aligned}
$$

where $D_{0}$ is the quasi-static displacement that would ultimately be achieved if the burst duration were infinite, $t_{o f f}$ is the time point when HIFU was switched off, $\tau_{\text {rise }}$ and $\tau_{\text {fall }}$ are the rise and fall time constants of tissue, respectively. Values of $3.2 \mathrm{~ms}$ and $5.5 \mathrm{~ms}$ were used based on papers by Huang et al. (24) and Kaye et al. (11). T1 =700 ms and T2 $=35 \mathrm{~ms}$ of muscle were used in the simulation.

\section{Ex vivo experiment setup}

All experiments were conducted on a 3.0T MR system (uMR790, Shanghai United Imaging Healthcare, Shanghai, China) with a gradient system capable of producing a maximum amplitude of $100 \mathrm{mT} / \mathrm{m}$. The experiments were carried out in three ex vivo porcine muscle samples and one ex vivo porcine brain sample that were bought the day of the experiment. The ex vivo porcine brain was fixed in $1.2 \%$ (w/v \%) agar gel phantom. All samples were immersed in degassed water, sealed in a vacuum chamber of $0.08 \mathrm{MPa}$ for half an hour, and then kept in the scanner room for another half an hour before the experiment. The start temperature of the samples was assumed to be the room temperature, which was $22{ }^{\circ} \mathrm{C}$. An MRI compatible HIFU transducer (0.8 MHz, Imasonic, Besançon, France) with a geometric focal distance of $100 \mathrm{~mm}$ (Figure 2) was used to generate HIFU pulses. Unbalanced gradients were added in the SS direction to encode the displacement-induced phase change in the coronal plane. The imaging parameters of the cES STARFI sequence were the following: TE/ $\mathrm{TR}=6.4 / 14.3 \mathrm{~ms}, \mathrm{FA}=10^{\circ}$, bandwidth $=500 \mathrm{~Hz} /$ pixel, resolution $=2.0 \times 2.0 \times 5.0 \mathrm{~mm}^{3}$, and matrix size $=128 \times 128$. An amplitude of the DEG lobe in the range of $25-60 \mathrm{mT} / \mathrm{m}$ and a duration in the range of $3-15 \mathrm{~ms}$ have been previously reported to generate good ARFI contrast and temperature quantification (10-14). In our experiment, the amplitude of gradients $\mathrm{C}$ and $\mathrm{D}$ were set to $50 \mathrm{mT} / \mathrm{m}$, the slew rate was set to $76.92 \mathrm{mT} / \mathrm{m} \cdot \mathrm{ms}$, and the duration was set to $4.53 \mathrm{~ms}$. Additionally, $M_{c}=-103.36 \mathrm{mT} / \mathrm{m} \cdot \mathrm{ms}$ and $M_{D}$ $=193.72 \mathrm{mT} / \mathrm{m} \cdot \mathrm{ms}$. The total acquisition time for a pair of images was $2 \times 1.83=3.66 \mathrm{~s}$. The imaging parameters for the spGRE STARFI sequence were almost the same as those for cES STARFI, except that TE/TR $=13.3 / 16.9 \mathrm{~ms}$ and $M_{c}=-M_{D}=-193.72 \mathrm{mT} / \mathrm{m} \cdot \mathrm{ms}$. The duration of gradients $\mathrm{C}$ and D was $4.53 \mathrm{~ms}$, and the total time of bipolar DEG was $9.06 \mathrm{~ms}$. Therefore, the minimum TE/TR of spGRE STARFI was longer than that of cES STARFI. The total acquisition time for a pair of images was $2 \times 2.16=4.32 \mathrm{~s}$. An 8 -channel small flex coil was put on top of the water tank for signal detection. The HIFU pulse was triggered by the transistor-transistor logic (TTL) signal synchronized to gradient D. The duration of the HIFU pulse was $4.53 \mathrm{~ms}$ for both sequences. The positive acoustic pressure at the focal point was $7.36 \mathrm{MPa}$ in most of the muscle samples and $3.62 \mathrm{MPa}$ in the brain sample. All experiments were performed three times in each sample for statistical analyses. 


\section{Optimal trigger delay}

The optimal trigger delay in ex vivo porcine muscle was experimentally verified. The $t_{\text {offset }}$ between the start time point of HIFU and the DEG was adjusted from -4 to $2 \mathrm{~ms}$ by $1 \mathrm{~ms}$ intervals. The optimal $t_{\text {offset }}$ was selected when the displacement-induced phase change that averaged over 5 pixels surrounding the focal spot reached the maximum value. The optimal trigger delays of cES STARFI and spGRE STARFI were used in all of the following experiments.

\section{Influence of acoustic pressure on displacement-induced phase change}

The acoustic pressure was firstly measured with a $0.2 \mathrm{~mm}$ needle hydrophone (Precision Acoustic Ltd., Dorset, UK) in water from 3.62 to $6.21 \mathrm{MPa}$. To avoid damaging the hydrophone, acoustic pressure above $6.21 \mathrm{MPa}$ was estimated by extrapolating the linear model between the input electric power and acoustic pressure instead of using the hydrophone directly. The influence of acoustic pressure on displacement-induced phase change was tested on porcine muscle samples; all other parameters were kept constant. The maximum displacement-induced phase changes of cES STARFI and spGRE STARFI in the focus were calculated under each acoustic pressure.

\section{Measurement uncertainty and differences}

The uncertainty and heating experiments were carried out in ex vivo porcine muscle and ex vivo porcine brain samples. The uncertainty experiments were carried out with the HIFU pulse OFF. The cES STARFI and spGRE STARFI sequences were scanned continuously for 60 pairs, and the temporal standard deviation of the displacement-induced phase change and the temperature change on a voxel-byvoxel basis were evaluated across all images in the time series to find the measurement uncertainties. The cES STARFI was then compared to the spGRE STARFI with the HIFU pulse ON. The optimal trigger delays for the porcine brain sample, $t_{\text {offset }}=-2 \mathrm{~ms}$ for cES STARFI and $t_{\text {offset }}=-1 \mathrm{~ms}$ for spGRE STARFI, were determined by the above-described experiment. The HIFU pulse ON experiment was repeated three times for each sample, and the time interval between each experiment was 10 minutes to ensure the sample had cooled to room temperature. $\mathrm{TE} / \mathrm{TR}=6.4 / 16.9 \mathrm{~ms}$ for cES STARFI was used instead of the minimum TR to ensure the same HIFU duty cycle of $13.4 \%$ for comparison. The DEG moment was kept constant between the two sequences, and the acquisition time for a pair of images was $4.32 \mathrm{~s}$. The HIFU pulse was triggered $\mathrm{ON}$ from pair \#5 to $\# 12$, and the cooling process was also recorded from pair $\# 13$ to \#60. The total measurement time was $259.58 \mathrm{~s}$, and the total HIFU ON time was $34.56 \mathrm{~s} \times 13.4 \%$, or $4.64 \mathrm{~s}$. The displacement-induced phase-to-noise ratio $\left(\mathrm{PNR}_{\mathrm{d}}\right)$ was defined as the maximum phase change at the focus divided by the standard deviation of the phase in a background region of interest (ROI) far from the focus. The $\mathrm{PNR}_{d}$ of the two sequences from pair \#5 to \#12 across three measurements was statistically compared by the Student's $t$-test.

\section{Results}

\section{Bloch simulation}

Figure 3 shows the Bloch simulation results. The phase sensitivities $\zeta$ to displacement of cES STRAFI and spGRE STRAFI are shown in Figure $3 A, B$. The results demonstrated that, for the spGRE sequence, the phase sensitivity to displacement $\zeta$ was around 1 ; this was consistent with our assumptions. The phase sensitivity to displacement $\zeta$ of the cES sequence was always more than 1. The phase sensitivity of cES STARFI became higher with prolonged T1. For the ex vivo porcine muscle samples (T1/T2 $=700 / 35 \mathrm{~ms}$ ) and ex vivo porcine brain sample (T1/ $\mathrm{T} 2=900 / 65 \mathrm{~ms}$ ), the phase sensitivities to displacement $\zeta$ of cES STARFI were 1.82 and 1.96 times that of spGRE STARFI, respectively, in the simulation. Figure $3 D$ compares the phase evolution of the $\mathrm{cES}$ sequence with and without HIFU pulses in tissues with T1/T2 $=700 / 35,900 / 65$, and $1,500 / 35 \mathrm{~ms}$, respectively. When HIFU was completely OFF, the phases of both echoes were always $-\pi / 2$, which showed that the phases between the two echoes were consistent. When HIFU was interleaved ON, it was found that displacement introduced additional phase changes into both echoes, producing different effects. Comparing tissues with different T1s (700 and 1,500 ms), the phases between Echo 2 and Echo 1 showed a greater difference with a longer T1. Figure $3 E$ compares the displacement-induced phase change of cES STARFI and spGRE STARFI according to Eq. [6] and Eq. [7]. The phase changes of cES STARFI were always larger than those of spGRE STARFI. The effect of displacement-induced phase change on the calculation of temperature was simulated in Figure 3C. For the most 


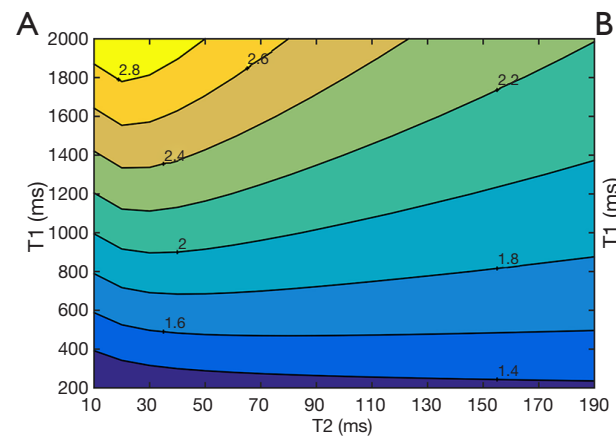

D

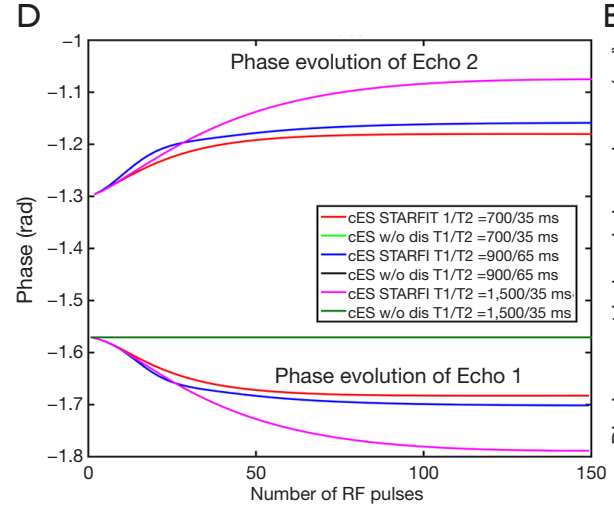

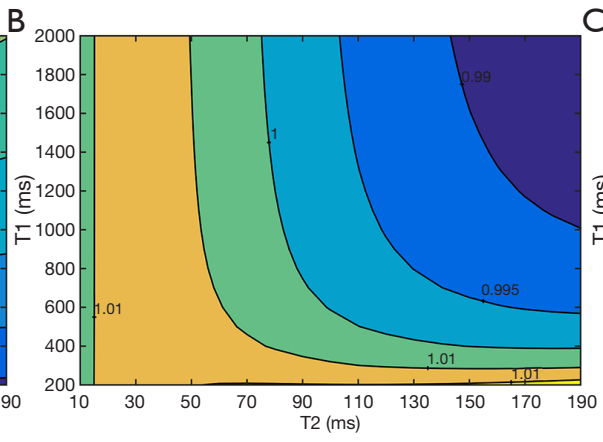
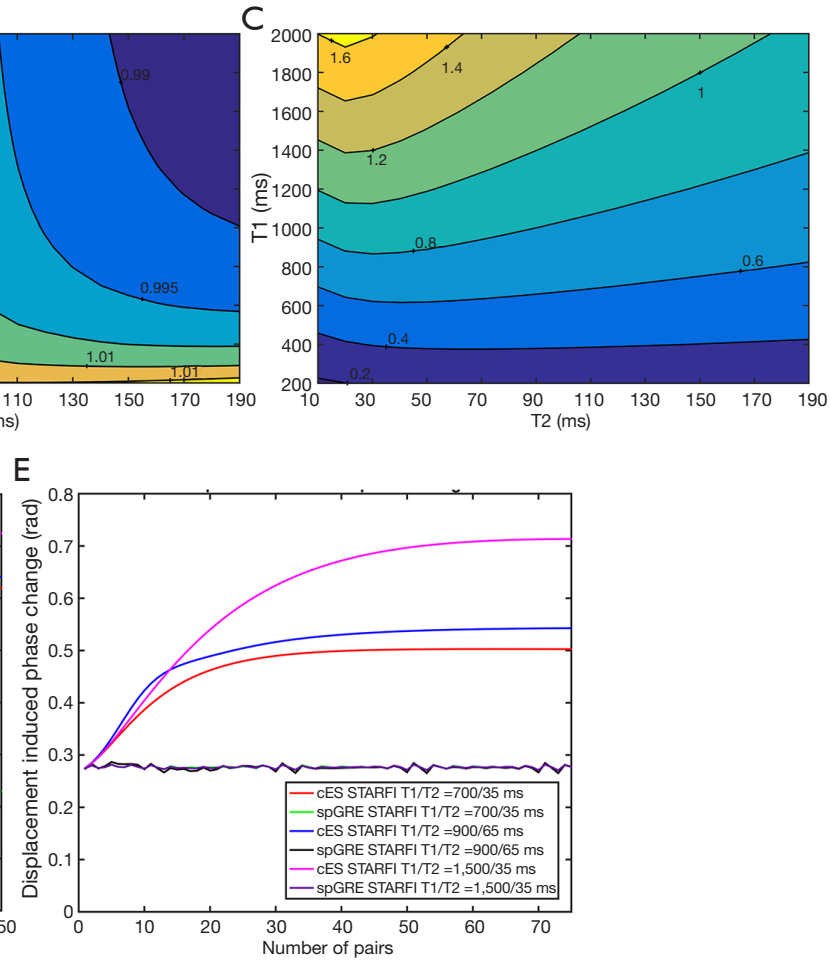

Figure 3 Bloch simulation of T1/T2 influence on the phase sensitivity to displacement $\zeta$ of (A) cES STARFI and (B) spGRE STARFI; (C) the impact of the displacement-induced phase change on temperature calculation; (D) the phase evolution of the cES STARFI signal with different T1/T2 combinations. The cES STARFI was compared to the cES signal without displacement (cES w/o dis). cES signals w/o dis of T1/T2 $=700 / 35,900 / 65$, and 1,500/35 ms, overlapped with each other; (E) the displacement-induced phase change evolution of cES STARFI and spGRE STARFI according to Eq. [6] and Eq. [7]. cES, coherent echo-shifted; spGRE, spoiled gradient echo.

common T1 (500-1,500 ms) and T2 (30-1,200 ms), the temperature bias from HIFU-induced displacement was around $0.6-1.2^{\circ} \mathrm{C}$.

\section{Optimal trigger delay}

The MR-ARFI results from muscle sample 1 are shown in Figure 4, and the results from all three muscle samples are summarized in Table 1. When the input HIFU power and duration were kept constant, the $t_{\text {offset }}$ between the start time point of HIFU and the DEG had a significant influence on the displacement-induced phase change of both $\mathrm{cES}$ STARFI and spGRE STARFI. Figure $4 A$ shows that the simulated and experimental displacement-induced phase changes. The results were normalized by the maximum phase change of spGRE STARFI occurring at $t_{\text {offse }}=-1 \mathrm{~ms}$. Except for muscle sample 3, all maximum displacement-induced phase changes of cES STARFI occurred at $t_{\text {offset }}=-2 \mathrm{~ms}$. The optimal toffset for cES STARFI shifted to $-3 \mathrm{~ms}$ in muscle sample 3. All maximum displacement-induced phase changes of spGRE STARFI occurred at $t_{\text {offset }}=-1 \mathrm{~ms}$. These trends were in good agreement across the simulation predictions and experiments. However, the differences between the maximum displacement-induced phase changes of cES STARFI and spGRE STARFI were slightly larger in the simulation compared with the experiments. Figure $4 B$ presents the displacement-induced phase change maps from muscle sample 1. The focus of spGRE STARFI could hardly be distinguished from the surrounding regions in the phase map at $t_{\text {offset }}=-4,-3$, and $2 \mathrm{~ms}$, yet they were still observable in the map based on cES STARFI. In all the following experiments, values of $t_{\text {offset }}=-2 \mathrm{~ms}$ for $\mathrm{cES}$ STARFI and $t_{\text {offset }}=-1 \mathrm{~ms}$ for spGRE STARFI were used.

\section{Influence of acoustic pressure on displacement-induced phase change}

The displacement-induced phase changes detected by cES 


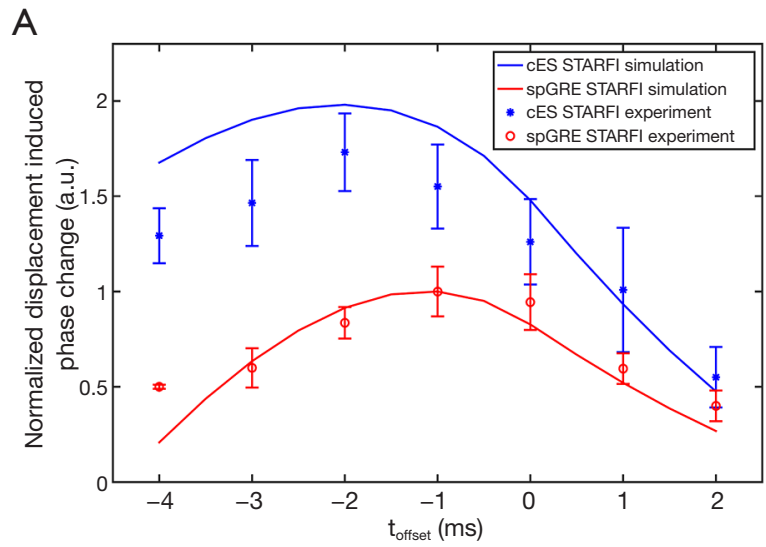

B

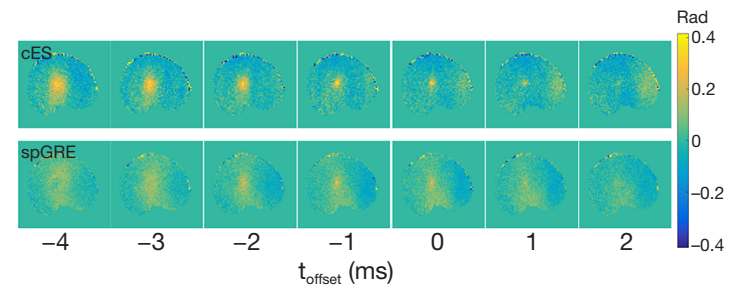

Figure 4 Influence of $t_{\text {offise }}$ on (A) the simulated and experimental displacement-induced phase change. The displacement-induced phase change was normalized by the maximum phase change of spGRE STARFI occurring at $t_{\text {offset }}=-1 \mathrm{~ms}$. T1/T2 $=700 / 25 \mathrm{~ms}$, $\tau_{\text {rise }}=3.2 \mathrm{~ms}$, and $\tau_{\text {fall }}=5.5 \mathrm{~ms}$ were used to model the displacementinduced phase change based on Eq. [9] and Eq. [10]; (B) the corresponding experimental displacement-induced phase maps. cES, coherent echo-shifted; spGRE, spoiled gradient echo.

STARFI and spGRE STARFI were compared by linearly increasing HIFU pressure. The fitted results from muscle sample 1 are shown in Figure 5A. The lowest acoustic pressure started from $3.62 \mathrm{MPa}$, under which the focus could be still observed in the displacement-induced phase map of cES STARFI, but could be hardly distinguished from that of spGRE (Figure $5 B$ ). While the measured maximum displacement-induced phase change was linearly increased with acoustic pressure for both sequences, their regression slopes were different. In all three muscle samples, in the case of cES STARFI, the slopes were 0.079, 0.079, and $0.047 \mathrm{rad} / \mathrm{MPa}$, respectively; the slopes of spGRE STARFI were only $0.047,0.052$, and $0.027 \mathrm{rad} / \mathrm{MPa}$, respectively.

\section{Measurement differences and uncertainties}

To compare the measurement differences and uncertainties of both sequences, the TR of cES STARFI was lengthened to $16.9 \mathrm{~ms}$, ensuring the HIFU duty cycles of these two sequences were both $13.4 \%$. Figure 6 shows the uncertainty maps of cES STARFI and spGRE STARFI in the muscle samples with HIFU OFF over the 60 measurements. The displacement-induced phase uncertainty of cES STARFI (Figure 6A) was larger than that of spGRE STARFI (Figure $6 B)$. The uncertainties of the displacement-induced phase change over the ROI were $0.078 \pm 0.0092 \mathrm{rad}$ for cES STARFI and $0.045 \pm 0.0059$ rad for spGRE STARFI. However, the temperature uncertainty of cES STARFI (Figure $6 C$ ) and spGRE STARFI (Figure 6D) were similar; the uncertainties were $0.30 \pm 0.038^{\circ} \mathrm{C}$ for cES STARFI and $0.26 \pm 0.047^{\circ} \mathrm{C}$ for spGRE STARFI, respectively.

Figure 7 displays a comparison of the displacementinduced phase and temperature monitoring results in muscle sample 1 acquired by cES STARFI and spGRE STARFI. Figure $7 A$ shows that the displacement-induced phase change based on cES STARFI was always larger than that of spGRE STARFI. Figure $7 B$ presents the temperature curves throughout the heating and cooling processes. At the end of HIFU sonication, the maximum temperature rise from the hottest pixel was $13.44 \pm 0.22{ }^{\circ} \mathrm{C}$ across three measurements when calculated by cES STARFI, and $12.34 \pm 0.09{ }^{\circ} \mathrm{C}$ when calculated by spGRE STARFI. The temperature change and instantaneously acquired displacement-induced phase change curves showed a strong consistency between both sequences. Figure 7C,D show the displacement-induced phase and temperature maps acquired during HIFU ON and OFF. While the displacement-induced phase maps were almost the same, the temperature gradually increased and the heated region was slightly enlarged with time. The $\mathrm{PNR}_{d}$ of cES STARFI was $6.59 \pm 1.03$, whereas the $\mathrm{PNR}_{d}$ of spGRE STARFI was $6.63 \pm 1.15$. As shown by $t$-test, the $\mathrm{PNR}_{\mathrm{d}}$ of the two sequences were not significantly different in this experiment $(\mathrm{P}=0.87)$.

Figure $8 A, B, C, D$ show the uncertainty maps of $\mathrm{cES}$ STARFI and spGRE STARFI in the ex vivo porcine brain sample across the 60 measurements. The displacementinduced phase uncertainty of cES STARFI was still greater than that of spGRE STARFI. The average uncertainties of displacement-induced phase change were $0.033 \pm 0.0031 \mathrm{rad}$ for cES STARFI and $0.019 \pm 0.0019$ rad for spGRE STARFI in the ROI indicated in Figure $8 A$. However, the temperature uncertainty of cES STARFI improved $(\mathrm{P}<0.05)$ due to the longer $\mathrm{T} 2 *$ in brain tissue. The average uncertainties of temperature change were $0.12 \pm 0.012{ }^{\circ} \mathrm{C}$ for cES STARFI and $0.16 \pm 0.023{ }^{\circ} \mathrm{C}$ for spGRE STARFI. Figure $8 E, F, G, H$ show the displacement-induced phase and temperature 
Table 1 Comparison of cES STARFI and spGRE STARFI in all the three ex vivo muscle samples

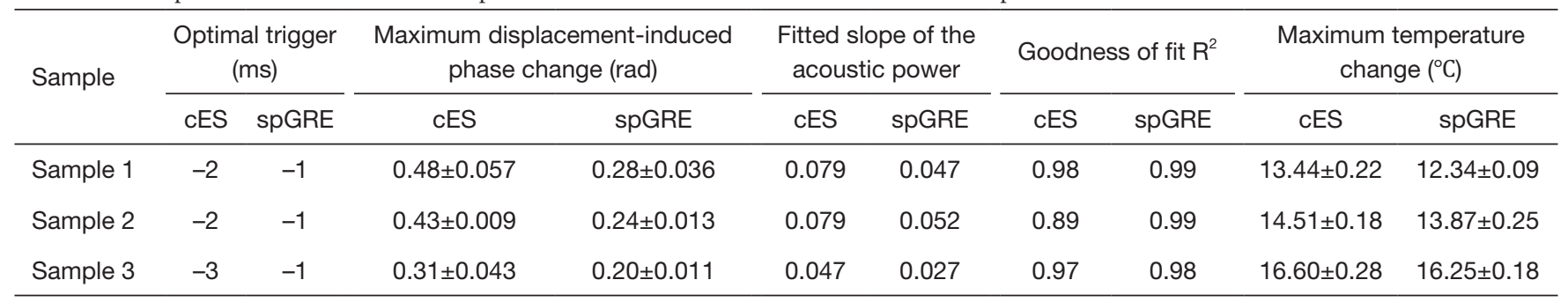

cES, coherent echo-shifted; spGRE, spoiled gradient echo.

\section{A}

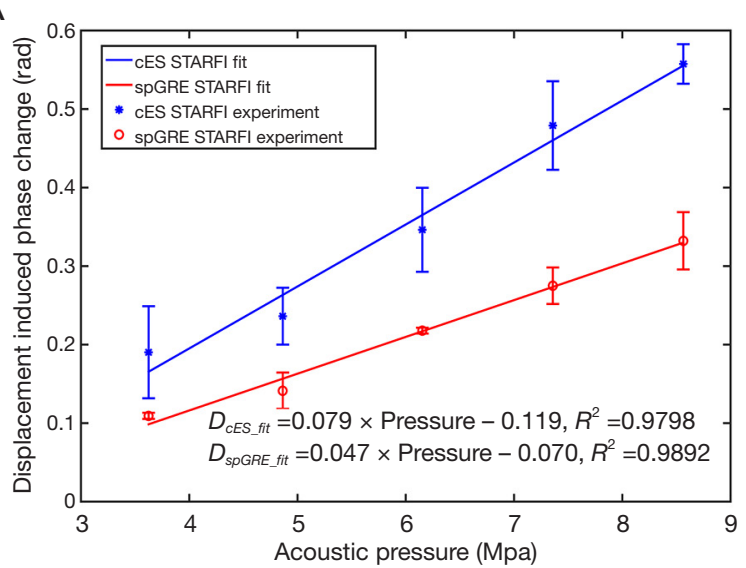

B

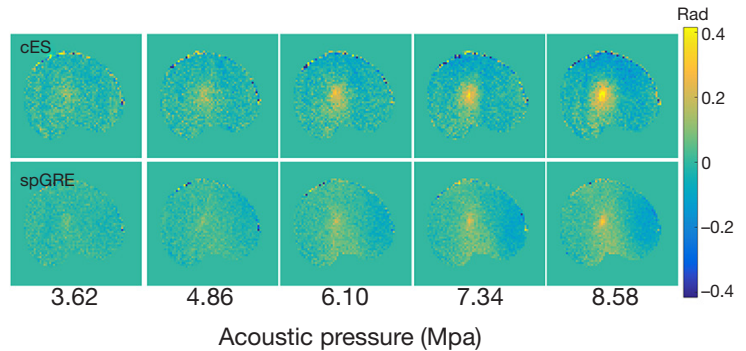

Figure 5 Influence of the input acoustic pressure on, (A) the maximum displacement-induced phase change at the focus, and (B) the corresponding displacement-induced phase change maps of cES STARFI and spGRE STARFI. cES, coherent echo-shifted; spGRE, spoiled gradient echo.

change during the heating experiment. The displacementinduced phase contrast of cES STARFI was more apparent than that of spGRE STARFI. The $\mathrm{PNR}_{\mathrm{d}}$ of cES STARFI was $14.16 \pm 1.81$, which is higher than that of spGRE STARFI $(11.93 \pm 1.68)(\mathrm{P}<0.05)$. The maximum temperature increases monitored by cES STARFI and spGRE STARFI were $4.41 \pm 0.02{ }^{\circ} \mathrm{C}$ and $3.93 \pm 0.05^{\circ} \mathrm{C}$, respectively; the difference was less than $0.5^{\circ} \mathrm{C}$.

\section{Discussion}

Simultaneous MR-ARFI and MRT imaging based on coherent ES sequence was evaluated in this study, and compared to the spGRE STARFI. The advantage of using an ES sequence to monitor the temperature change has been previously reported $(17,25)$; however, to our knowledge, few studies have examined the phase sensitivity to displacement of cES sequences.

cES sequence is suitable for focus localization for the following reasons. Firstly, cES showed a higher phase sensitivity to displacement than spGRE (Figure 3). This increased phase sensitivity to displacement was also verified by linearly increasing HIFU power. In Figure $5 B$, the small phase change induced by the lowest acoustic power was nearly indistinguishable from the background in the spGRE images, but, although there was more noise, it was still visible in cES images. The increased phase sensitivity method is attractive for use in transcranial focal localization as it is less demanding of the FUS input power. This can decrease the risk of damage to normal tissues. Another interesting feature of cES sequence is that the higher phase sensitivity to displacement was found to be related to the T1 value of the object in the Bloch simulation (Figure 3). We hypothesize that this increased displacement sensitivity comes from the stimulated echo pathways (26), which demonstrate T1 dependency. The phase contrast accumulated in the former TR intervals is stored as longitudinal magnetization (27) before another $\mathrm{RF}$ pulse reconverts them back to the transverse plane. The longitudinal pathway exhibits T1-dependent exponential decay during their stay along the longitudinal axis (28). Therefore, the longer the T1, the more longitudinal component is preserved, contributing to increased sensitivity to displacement in the final signal.

Secondly, cES STARFI is more time efficient. With the same DEG moment and other imaging parameters, 

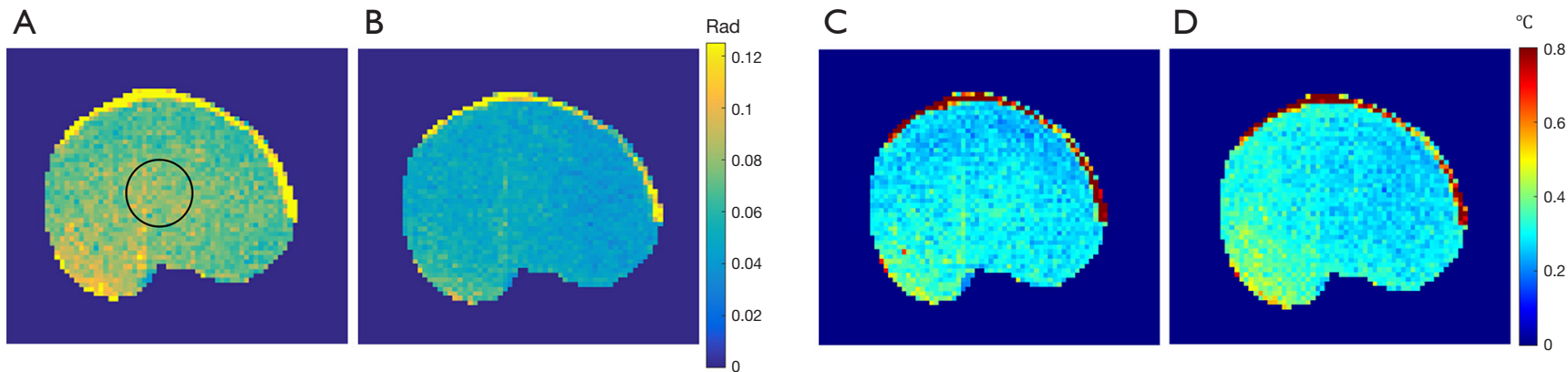

Figure 6 The standard deviation maps of the displacement-induced phase and the temperature change of (A,C) cES STARFI and (B,D) spGRE STARFI over a total of 60 measurements. The standard deviations were averaged over a ROI indicated by the black circle in (A) to evaluate measurement uncertainty. cES, coherent echo-shifted; spGRE, spoiled gradient echo; ROI, region of interest.
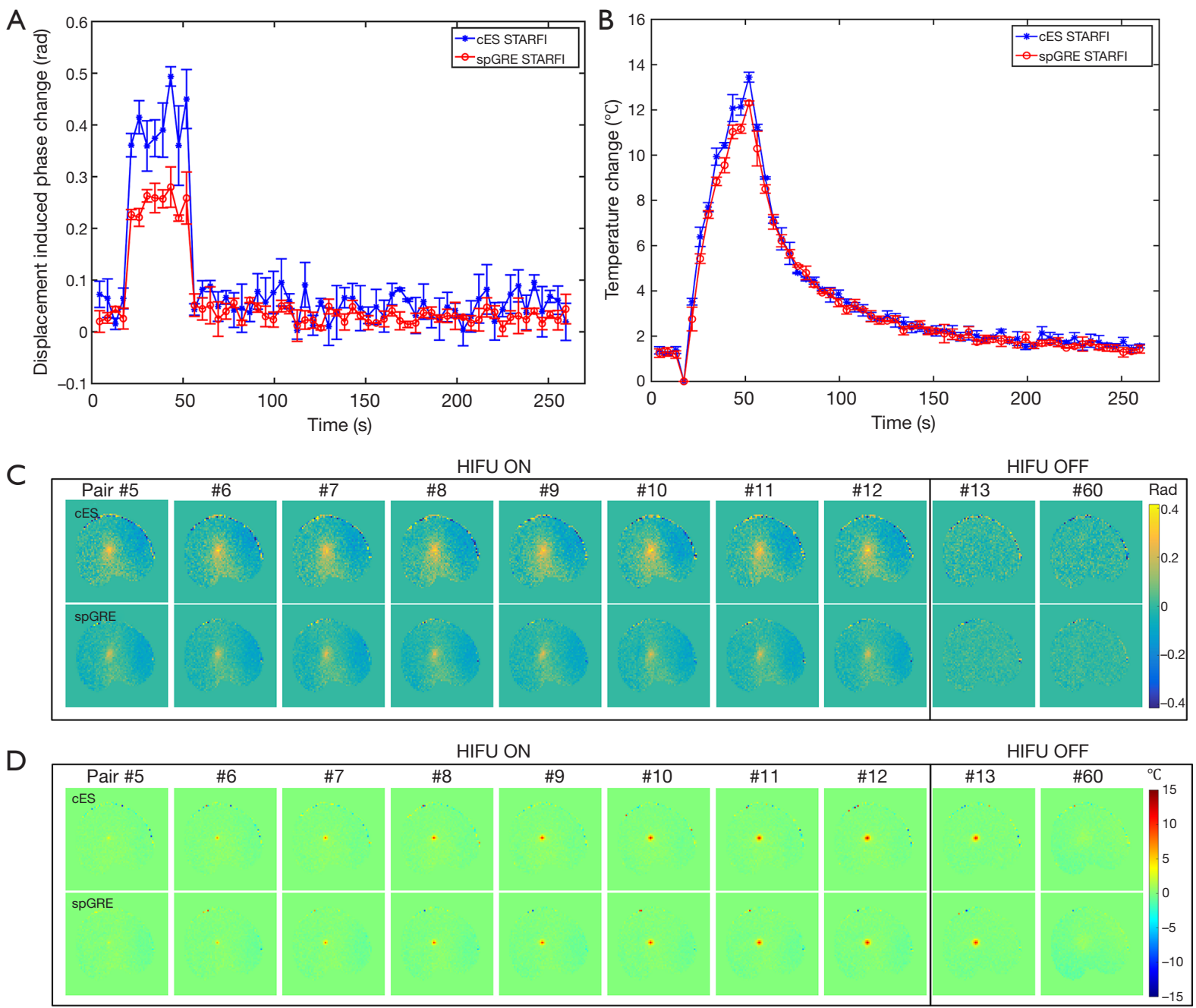

Figure 7 (A) The displacement-induced phase change and (B) the temperature change curves of cES STARFI and spGRE STARFI in muscle sample 1; (C) the displacement-induced phase change and (D) the temperature maps acquired when HIFU was ON (from pairs \#5 to \#12). Two images, one when HIFU was OFF (pair \#13) and one at the end of the acquisition (pair \#60), are also included. cES, coherent echo-shifted; spGRE, spoiled gradient echo; HIFU, high-intensity focused ultrasound. 


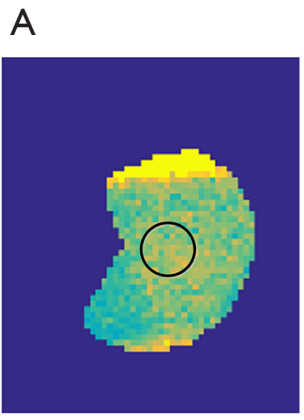

E

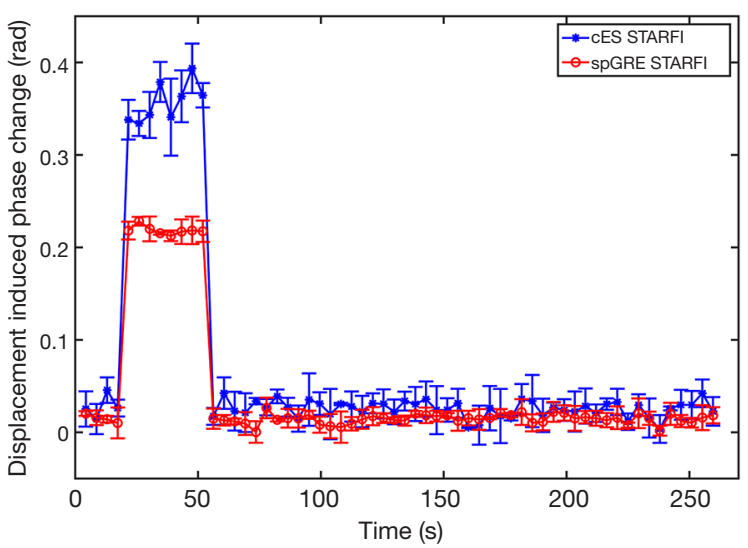

B

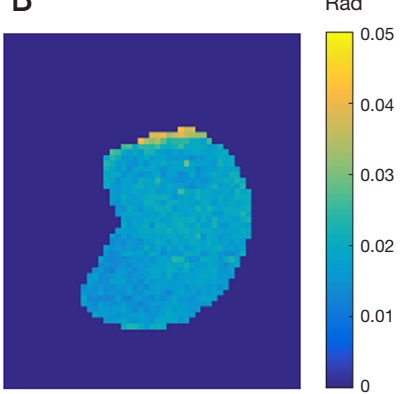

$\mathrm{F}$
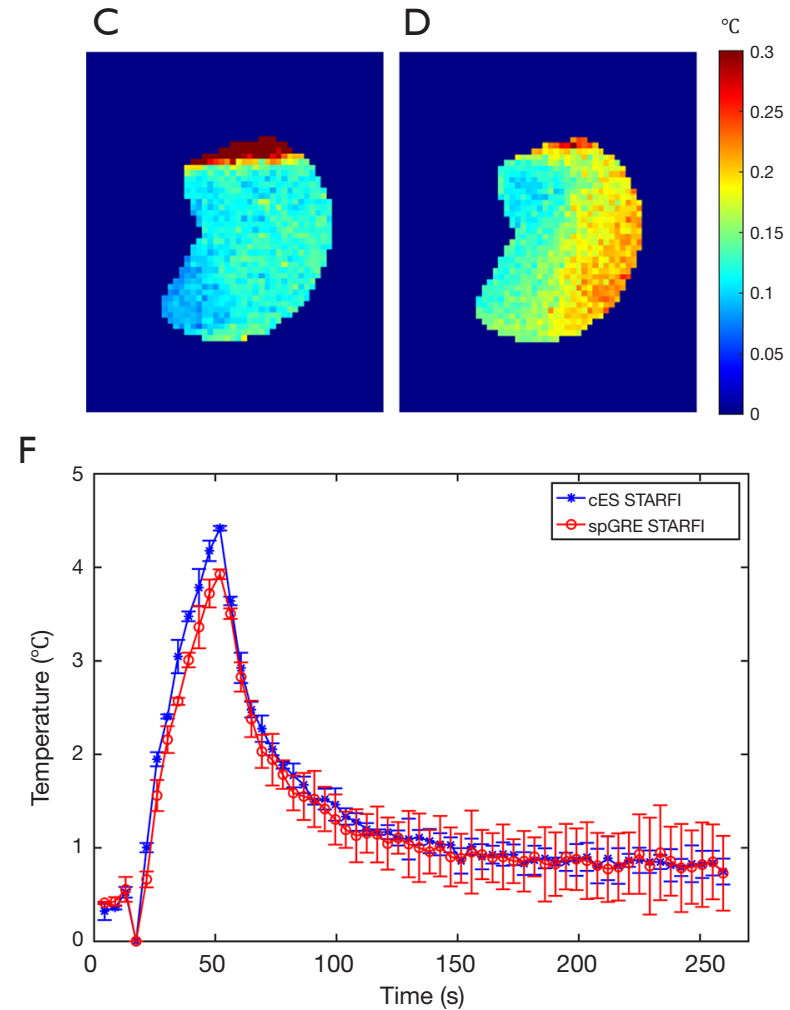

G
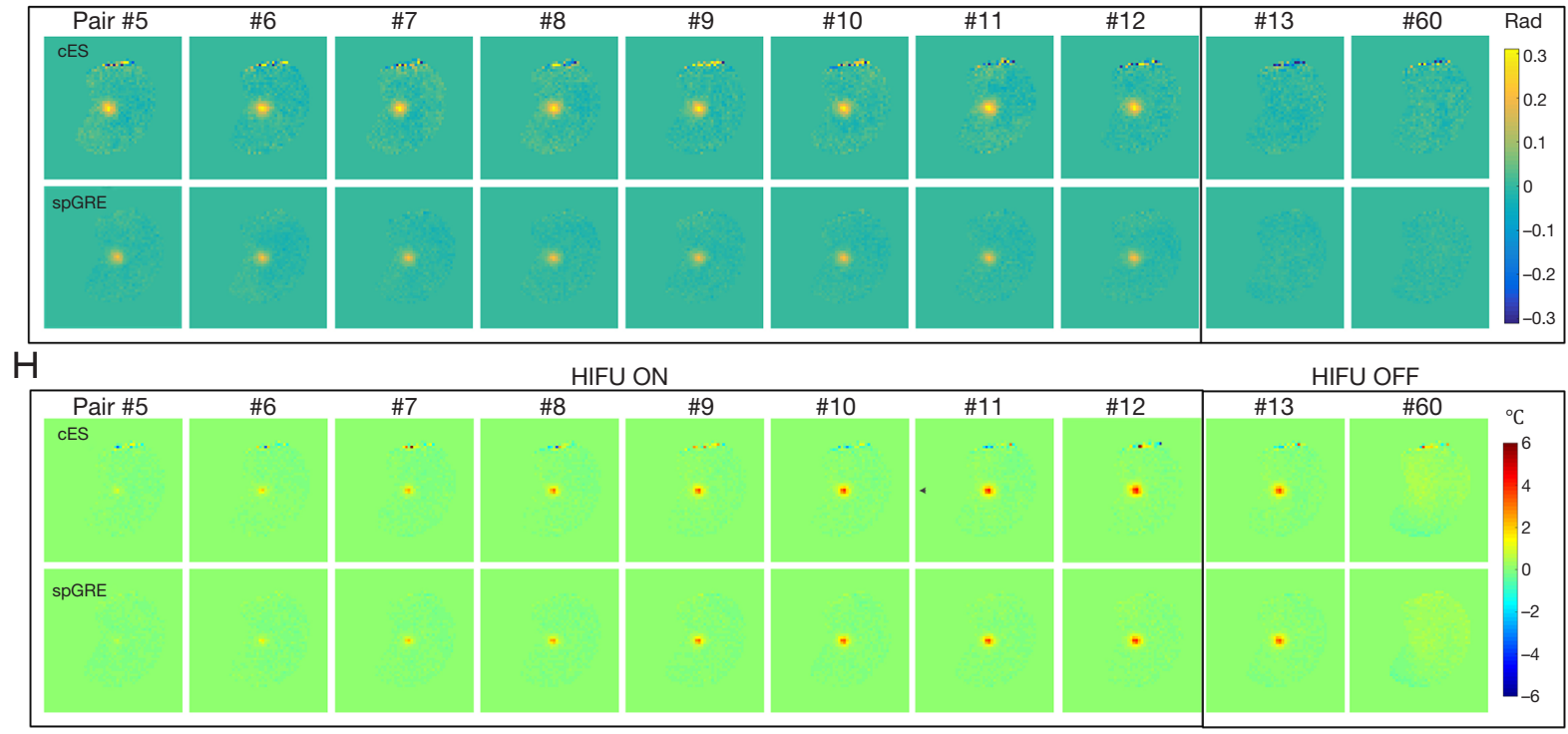

Figure 8 The standard deviation of the displacement-induced phase change and the temperature change of (A,C) cES STARFI and (B,D) spGRE STARFI in ex vivo porcine brain when HIFU was completely OFF. The standard deviations were averaged over the ROI indicated by the black circle in (A); (E) the displacement-induced phase change and (F) the temperature change curves of cES STARFI and spGRE STARFI; $(\mathrm{G})$ the displacement-induced phase and $(\mathrm{H})$ the temperature maps acquired when HIFU was ON (from pair \#5 to \#12), when HIFU was OFF (pair \#13), and at the end of acquisition (pair \#60). cES, coherent echo-shifted; spGRE, spoiled gradient echo; HIFU, highintensity focused ultrasound; ROI, region of interest. 
the required TR of the cES STARFI sequence was always shorter than that of the spGRE STARFI. Both sequences had the same DEG moment (MD $=193.72 \mathrm{mT} / \mathrm{m} \cdot \mathrm{ms})$, and the minimum TR of cES STARFI was $15.38 \%$ shorter than that of spGRE STARFI.

The ex vivo muscle experiment (Figure 4) demonstrated that the differences between the maximum displacementinduced phase changes of cES STARFI and spGRE STARFI were smaller than those in the simulation, with $\mathrm{T} 1 / \mathrm{T} 2=700 / 35 \mathrm{~ms}$. We suspect this is due to the diffusion effect, which was not considered in the Bloch simulation. As mentioned above, the higher phase sensitivity to displacement was influenced by longitudinal magnetization. However, "stored" longitudinal magnetizations are subject to the noticeable diffusion attenuation (29). Additionally, the rise and fall time constants in the in vivo rabbit thigh muscle were used in the present study's simulation. These values could be different from those of the ex vivo porcine muscle used in the experiment.

The displacement-induced phase uncertainty of cES STARFI was higher than that of spGRE STARFI (Figure $6 A, B$ and Figure $8 A, B)$. This was predictable, as the image signal-to-noise ratio (SNR) of the cES sequence was lower than that of the spGRE sequence. The standard deviation $\sigma_{\varphi}$ was determined by magnitude image SNR as $\sigma_{\varphi} \sim 1 / \mathrm{SNR}$ (30). However, from the contrast-to-noise ratio view, the $\mathrm{PNR}_{\mathrm{d}}$ of cES STARFI was nearly the same as that of spGRE STARFI due to the increased phase sensitivity in $e x$ vivo porcine muscle. In the ex vivo brain sample, the $\mathrm{PNR}_{\mathrm{d}}$ of cES STARFI was improved compared with that of spGRE STRAFI due to the even larger phase sensitivity. The temperature uncertainty of cES STARFI in ex vivo porcine muscle was slightly greater than that of spGRE STARFI (Figure 6C,D). This is because, as the $\mathrm{T}^{*}$ in muscle is quite short, the increased temperature sensitivity that resulted from the larger TEeff was canceled out by the SNR loss (25). However, in the ex vivo brain sample, which had a longer $\mathrm{T} 2 / \mathrm{T} 2 *$, the lengthened effective TE led to higher temperature precision with cES STARFI than with spGRE STARFI (Figure 8C,D).

The displacement-induced phase change was expected to be slightly larger in brain tissue (1.96 times that of spGRE STARFI) than in muscle (1.82 times that of spGRE STARFI) due to the longer T1 in white matter. However, there were no apparent differences between Figure $7 A$ and Figure $8 E$. This might be because the diffusion effect and the elasticity of tissue was not considered in the simulation, which might have caused the difference to be overestimated.
The simultaneous ARFI and temperature results shown in Figure 7 and Figure 8E,F,G,H confirm that using cES STARFI to monitor HIFU treatment is feasible. Madore et al. (21) studied the application of multiple pathway sequences for MRT and concluded that the effective TE for any given pathway was $p T R+T E$, where $p$ is the pathway index. For the cES sequence, $\mathrm{p}$ is equal to 1 . Therefore, the effective TE is equal to TR + TE. However, when the HIFU pulses were interleaved ON/OFF, the displacement introduced an additional phase in Echo 1, which resulted in temperature overestimation in the experiments. Based on the current simulation parameters, this temperature bias was relatively small for most tissues. To eliminate this temperature bias, the image taken immediately after HIFU pulse $\mathrm{ON}$ can be used as the reference in Eq. [3] in case the temperature change in the first image right after HIFU pulse $\mathrm{ON}$ is negligible.

The biggest limitation of cES STARFI was that the displacement could not be quantitatively evaluated. This is because the phase sensitivity $\zeta$ depends on the T1/T2 of the tissue. Therefore, only displacement-induced phase change maps were generated in our study. The SNR loss due to heavy $\mathrm{T} 2{ }^{*}$ weighting in turn caused greater measurement uncertainty. However, heavy T2* weighting also made the sequence sensitive to susceptibility effects and micro bleeding caused by HIFU treatment, which may be another advantage of using the proposed sequence for treatment monitoring.

\section{Conclusions}

In this study, a new method of simultaneous MR-ARFI and temperature monitoring of HIFU treatment based on a coherent cES sequence was explored and comprehensively compared to spGRE STARFI. The displacement encoding of the cES sequence was more time efficient than spGRE STARFI. Increased phase sensitivity to the displacement of cES STARFI was observed in Bloch simulations and ex vivo experiments. The $\mathrm{PNR}_{\mathrm{d}}$ and temperature uncertainty of the cES STARFI were improved compared with those of spGRE STARFI in an ex vivo brain sample. We conclude that the cES sequence provided an effective method of visualizing the focal spot, and may be a desirable alternative to the use of spGRE STARFI during pulsed HIFU applications.

\section{Acknowledgments}

Funding: This research was supported by the National 
Natural Science Foundation of China (no. 81527901 and 11504401), the Shenzhen Key Laboratory of Ultrasound Imaging and Therapy (no. ZDSYS 201802061806314), the Guangdong Grant for "Key Technologies for Treatment of Brain Disorders" (no. 2018B030332001), the Key Laboratory for Magnetic Resonance and Multimodality Imaging of Guangdong Province (no. 2014B030301013), the Shenzhen Double Chain Grant (2018) 256, the Scientific Instrument Innovation Team of the Chinese Academy of Sciences (no. GJJSTD20180002), and the Chinese Postdoctoral Science Foundation (no. 2019M650220).

\section{Footnote}

Conflicts of Interest: All authors have completed the ICMJE uniform disclosure form (available at http://dx.doi. org/10.21037/qims-20-274). DL serves as an unpaid editorial board member of Quantitative Imaging in Medicine and Surgery. The other authors have no conflicts of interest to declare.

Ethical Statement: Ethical approval/informed consent is not applicable, because all experiments were carried out in ex vivo tissue bought at the local grocery store.

Open Access Statement: This is an Open Access article distributed in accordance with the Creative Commons Attribution-NonCommercial-NoDerivs 4.0 International License (CC BY-NC-ND 4.0), which permits the noncommercial replication and distribution of the article with the strict proviso that no changes or edits are made and the original work is properly cited (including links to both the formal publication through the relevant DOI and the license). See: https://creativecommons.org/licenses/by-nc-nd/4.0/.

\section{References}

1. Voogt MJ, Trillaud H, Kim YS, Mali WP Th M, Barkhausen J, Bartels LW, Deckers R, Frulio N, Rhim H, Lim HK, Eckey T, Nieminen HJ, Mougenot C, Keserci B, Soini J, Vaara T, Köhler MO, Sokka S, van den Bosch MAA J. Volumetric feedback ablation of uterine fibroids using magnetic resonance-guided high intensity focused ultrasound therapy. Eur Radiol 2012;22:411-7.

2. Blana A, Walter B, Bogenhofer S, Wieland WF. Highintensity focused ultrasound for the treatment of localized prostate cancer: 5-year experience. Urology
2004;63:297-300.

3. Elias WJ, Huss D, Voss T, Loomba J, Khaled M, Zadicario E, Frysinger RC, Sperling SA, Wylie S, Monteith SJ, Druzgal J, Shah BB, Harrison M, Wintermark M. A pilot study of focused ultrasound thalamotomy for essential tremor. N Engl J Med 2013;369:640-8.

4. Zhu M, Sun Z, Ng CK. Image-guided thermal ablation with MR-based thermometry. Quant Imaging Med Surg 2017;7:356-68.

5. McDannold N, Maier SE. Magnetic resoance acoustic radiation force imaging. Med Phys 2008;35:3748-58.

6. Radicke M, Engelbertz A, Habenstein B, Lewerenz M, Oehms O, Trautner P, Weber B, Wrede S, Maier K. New image contrast method in magnetic resonance imaging via ultrasound. Hyperfine Interactions 2008;181:21-6.

7. Larrat B, Pernot M, Montaldo G, Fink M, Tanter M. Energy-based adaptive focusing: optimal ultrasonic focusing using magnetic resonance guidance. Roma: 2009 IEEE International Ultrasonics Symposium, 2009:57-60.

8. Hertzberg Y, Volovick A, Zur Y, Medan Y, Vitek S, Navon G. Ultrasound focusing using magnetic resonance acoustic radiation force imaging: application to ultrasound transcranial therapy. Med Phys 2010;37:2934-42.

9. Kaye EA, Chen J, Pauly KB. Rapid MR-ARFI method for focal spot localization during focused ultrasound therapy. Magn Reson Med 2011;65:738-43.

10. Auboiroux V, Viallon M, Roland J, Hyacinthe JN, Petrusca L, Morel DR, Goget T, Terraz S, Gross P, Becker CD, Salomir R. ARFI-prepared MRgHIFU in liver: Simultaneous mapping of ARFI-displacement and temperature elevation, using a fast GRE-EPI sequence. Magn Reson Med 2012;68:932-46.

11. Kaye EA, Pauly KB. Adapting MRI acoustic radiation force imaging for in vivo human brain focused ultrasound applications. Magn Reson Med 2013;69:724-33.

12. Mougenot C, Waspe A, Looi T, Drake JM. Variable ultrasound trigger delay for improved magnetic resonance acoustic radiation force imaging. Phys Med Biol 2016;61:712-27.

13. Bour P, Marquet F, Ozenne V, Toupin S, Dumont E, Aubry JF, Lepetit-Coiffe M, Quesson B. Real-time monitoring of tissue displacement and temperature changes during MRguided high intensity focused ultrasound. Magn Reson Med 2017;78:1911-21.

14. de Bever JT, Odeen H, Hofstetter LW, Parker DL. Simultaneous MR thermometry and acoustic radiation force imaging using interleaved acquisition. Magn Reson Med 2018;79:1515-24. 
15. Jolesz FA, Hynynen KH, MRI-guided focused ultrasound surgery. New York: Informa Healthcare, 2007; Chapter 2:15-7.

16. Zheng Y, Marx M, Miller GW, Butts Pauly K. High sensitivity MR acoustic radiation force imaging using transition band balanced steady-state free precession. Magn Reson Med 2018;79:1532-7.

17. Peng Y, Zou C, Qiao Y, Tie C, Wan Q, Jiang R, Cheng C, Liang D, Zheng H, Li F, Liu X. Fast MR thermometry using an echo-shifted sequence with simultaneous multislice imaging. MAGMA 2018;31:771-9.

18. Moonen CT, Liu G, van Gelderen P, Sobering G. A fast gradient-recalled MRI technique with increased sensitivity to dynamic susceptibility effects. Magn Reson Med 1992;26:184-9.

19. Qiao Y, Zou C, Cheng C, Wan Q, Tie C, Liang D, Zheng $\mathrm{H}$, Liu X, Chung Y. Diffusion effect on T2 relaxometry in triple-echo steady state free precession sequence. J Magn Reson 2018;292:25-35.

20. De Poorter J, De Wagter C, De Deene Y, Thomsen C, Ståhlberg F, Achten E. Noninvasive MRI thermometry with the proton resonance frequency (PRF) method: in vivo results in human muscle. Magn Reson Med 1995;33:74-81.

21. Madore B, Panych LP, Mei CS, Yuan J, Chu R. Multipathway sequences for MR thermometry. Magn Reson Med 2011;66:658-68.

22. Souchon R, Salomir R, Beuf O, Milot L, Grenier D, Lyonnet D, Chapelon JY, Rouvière O. Transient MR

Cite this article as: Qiao Y, Zou C, Cheng C, Tie C, Wan Q, Peng H, Liang D, Liu X, Zheng H. Simultaneous acoustic radiation force imaging and $M R$ thermometry based on a coherent echo-shifted sequence. Quant Imaging Med Surg 2020;10(9):1823-1836. doi: 10.21037/qims-20-274 elastography (t-MRE) using ultrasound radiation force: Theory, safety, and initial experiments in vitro. Magn Reson Med 2008;60:871-81.

23. Dadakova T, Krafft AJ, Özen AC, Bock M. Optimization of acoustic radiation force imaging: influence of timing parameters on sensitivity. Magn Reson Med 2018;79:981-6.

24. Huang Y, Curiel L, Kukic A, Plewes DB, Chopra R, Hynynen K. MR acoustic radiation force imaging: in vivo comparison to ultrasound motion tracking. Med Phys 2009;36:2016-20.

25. de Zwart JA, van Gelderen P, Kelly DJ, Moonen CTW. Fast magnetic-resonance temperature imaging. J Magn Reson B 1996;112:86-90.

26. Denolin V, Metens T. On the calculation and interpretation of signal intensity in echo-shifted sequences. Magn Reson Med 2004;51:123-34.

27. Haacke EM, Brown RW, Thompson MR, Venkatesan R. Magnetic resonance imaging: physical principles and sequence design. New York: Wiley-Liss, 1999; Chapter 18:488.

28. Weigel M. Extented phase graphs: dephasing, RF pulses, and echoes-pure and simple. J Magn Reson Imaging 2015;41:266-95.

29. Weigel M, Schwenk S, Kiselev VG, Scheffler K, Henning J. Extended phase graphs with anisotropic diffusion. J Magn Reson 2010;205:276-85.

30. Chen J, Watkins R, Pauly KB. Optimization of encoding gradients for MR-ARFI. Magn Reson Med 2010;63:1050-8. 